\title{
Replication study for the association of new meta-analysis-derived risk loci with susceptibility to type 2 diabetes in 6,244 Japanese individuals
}

\author{
S. Omori • Y. Tanaka $\cdot$ M. Horikoshi • A. Takahashi • \\ K. Hara • H. Hirose • A. Kashiwagi • K. Kaku • \\ R. Kawamori • T. Kadowaki • Y. Nakamura • S. Maeda
}

Received: 30 March 2009 /Accepted: 22 April 2009 /Published online: 20 May 2009

(C) Springer-Verlag 2009

\begin{abstract}
Aims/hypothesis Additional susceptibility loci for type 2 diabetes have been identified by a meta-analysis of genome-wide association studies (GWASs) in European populations. To examine further the roles of these new loci, we performed a replication study for the association of these single-nucleotide polymorphism (SNP) loci with the disease in three independent Japanese populations.

Methods We genotyped seven of the 11 SNPs that emerged in stage 2 of the meta-analysis for European GWASs (rs864745 in JAZF1, rs12779790 near CDC123/CAMK1D, rs7961581 near TSPAN8/LGR5, rs4607103 near ADAMTS9,
\end{abstract}

Electronic supplementary material The online version of this article (doi:10.1007/s00125-009-1397-5) contains supplementary material, which is available to authorised users.

S. Omori $\cdot$ S. Maeda $(\bowtie)$

Laboratory for Endocrinology and Metabolism,

RIKEN Center for Genomic Medicine,

1-7-22 Suehiro-cho, Tsurumi-ku,

Yokohama, Kanagawa 230-0045, Japan

e-mail: smaeda@src.riken.jp

S. Omori $\cdot$ Y. Tanaka

Department of Internal Medicine,

Division of Metabolism and Endocrinology,

St Marianna University School of Medicine,

Kawasaki, Kanagawa, Japan

M. Horikoshi $\cdot$ K. Hara $\cdot$ T. Kadowaki

Department of Metabolic Diseases, Graduate School of Medicine,

University of Tokyo,

Tokyo, Japan

A. Takahashi

Laboratory for Statistical Analysis,

RIKEN Center for Genomic Medicine,

Tokyo, Japan rs10923931 in NOTCH2, rs1153188 near DCD and rs9472138 near VEGFA) for three independent Japanese populations (first set, 1,630 type 2 diabetes patients vs 1,064 controls; second set, 1,272 type 2 diabetes patients vs 856 controls; third set, 486 type 2 diabetes patients vs 936 controls) using a TaqMan assay. The association of the SNP loci in each population was analysed using a logistic regression analysis, adjusting for age, sex and BMI, and the data were evaluated by a meta-analysis.

Results A meta-analysis for the three case-control studies identified a nominal association of rs864745 in JAZF1 with type 2 diabetes (OR 1.148, 95\% CI 1.034-1.275, $p=$

H. Hirose

Health Center, Keio University School of Medicine,

Tokyo, Japan

A. Kashiwagi

Department of Medicine, Shiga University of Medical Science,

Otsu, Shiga, Japan

K. Kaku

Division of Endocrinology and Metabolism,

Department of Internal Medicine, Kawasaki Medical School,

Kurashiki, Okayama, Japan

R. Kawamori

Department of Medicine, Metabolism and Endocrinology,

School of Medicine, Juntendo University,

Tokyo, Japan

Y. Nakamura

Laboratory of Molecular Medicine, Human Genome Center, Institute of Medical Science, University of Tokyo,

Tokyo, Japan 
0.0098 , corrected $p=0.069$ ). The association of other loci did not reach statistically significant levels (nominal $p>$ $0.05)$.

Conclusions/interpretation From these results the contribution of these seven loci in conferring susceptibility to type 2 diabetes is considered minor in the Japanese population, if they are present.

Keywords Association study · Japanese - SNP. Type 2 diabetes

$\begin{array}{ll}\text { Abbreviations } \\ \text { GWAS } & \text { Genome-wide association study } \\ \text { HWE } & \text { Hardy-Weinberg equilibrium } \\ \text { SNP } & \text { Single-nucleotide polymorphism }\end{array}$

\section{Introduction}

Type 2 diabetes affects more than 200 million individuals worldwide, and its prevalence is still progressively increasing in many countries, including Japan. The precise mechanisms underlying the development and progression of type 2 diabetes have not been elucidated yet, but a combination of multiple genetic and/or environmental factors might play important roles in the pathogenesis of the disease [1].

Following the discovery of the transcription factor 7-like 2 gene (TCF7L2) as a strong susceptibility gene for type 2 diabetes [2], genome-wide association studies (GWASs) conducted by several independent European and American research groups have identified multiple susceptibility loci for type 2 diabetes in populations of European descent [37]. Sladek et al. identified the haematopoietically expressed homeobox (HHEX) and solute carrier family 30 member 8 (SLC30A8) locus [3], and the Wellcome Trust Case Control Consortium (WTCCC)/United Kingdom Type 2 Diabetes (UKT2D), Diabetes Genetics Initiative (DGI) and Finland-United States Investigation of NIDDM Genetics (FUSION) groups identified the CDK5 regulatory subunit associated protein 1-like 1 (CDKAL1), cyclin-dependent kinase inhibitor-2A/B $(C D K N 2 A / B)$ and insulin-like growth factor 2 mRNA binding protein 2 (IGF2BP2) locus as susceptibility loci for type 2 diabetes in addition to TCF7L2, potassium inwardly rectifying channel subfamily $\mathrm{J}$ member 11 (KCNJ11) and peroxisome proliferatoractivated receptor gamma $(P P A R G)$ locus [4-6]. The associations of CDKAL1 and SLC30A8 with the disease were also identified by another GWAS conducted in the Icelandic population [7]. In addition, Japanese research groups, including ours, recently identified an association of the potassium voltage-gated channel KQT-like subfamily member 1 (KCNQ1) locus with type 2 diabetes in populations of East Asian descent [8, 9]. Because most of these loci have also been shown to be associated with type 2 diabetes in other ethnic populations [8-13], these loci could be considered as convincing susceptibility genes for type 2 diabetes across different ethnicities.

Recently, at least six additional susceptibility loci for type 2 diabetes were identified by a meta-analysis of GWASs and subsequent large-scale replication studies in populations of European descent [14]. Although these additional loci are also considered as susceptibility loci for the disease, it is necessary to evaluate the contributions of these loci in conferring susceptibility to type 2 diabetes in other ethnic populations; this is because it is well known that there are significant differences in the frequencies of some genetic variations among different ethnic groups [15-17].

The aim of the present study was to determine whether the variations identified by the meta-analysis of GWASs in European populations are associated with susceptibility to type 2 diabetes in the Japanese.

\section{Methods}

Participants, DNA preparation and genotyping For the RIKEN case-control study (first set), DNA samples were obtained from the peripheral blood samples of type 2 diabetes patients recruited from the outpatient clinics of the Shiga University of Medical Science and Kawasaki Medical School (Case 1; $n=1,630,978$ men and 652 women, Table 1) [10], We also examined 1,064 Control individuals who were enrolled from an annual health check conducted at either Juntendo University or Keio University (Control 1; $n=1,064,638$ men and 426 women, Table 1) [10].

For the University of Tokyo study (second set), we enrolled 1,272 patients with type 2 diabetes who visited the outpatient clinic of the Department of Metabolic Diseases, Graduate School of Medicine, University of Tokyo or the Hiroshima Atomic Bomb Casualty Council Health Management Center (Case 2; $n=1,272,765$ men and 507 women, Table 1) [11]. Exclusion criteria for cases were individuals with diabetes caused by: (1) liver dysfunction; (2) steroids and other drugs that might raise glucose levels; (3) malignancy; (4) monogenic disorder known to cause diabetes; and (5) positive for anti-GAD antibody. Control individuals were enrolled from an annual health check-up conducted at the Hiroshima Atomic Bomb Casualty Council Health Management Center (Control 2; $n=856$, 383 men and 473 women, Table 1) [11].

For the St Marianna University Study (third set), we further enrolled 486 patients with type 2 diabetes from the outpatient clinic of St Marianna University School of Medicine (Case 3; $n=486,288$ men and 198 women, Table 1). In this study, we used 936 individuals from the 
Table 1 Clinical characteristics of the participants

\begin{tabular}{|c|c|c|c|c|c|c|}
\hline \multirow[t]{2}{*}{ Characteristic } & \multicolumn{2}{|l|}{ Set 1} & \multicolumn{2}{|l|}{ Set 2} & \multicolumn{2}{|l|}{ Set 3} \\
\hline & Case 1 & Control 1 & Case 2 & Control 2 & Case 3 & Control 3 \\
\hline$n$ & 1,630 & 1,064 & 1,272 & 856 & 486 & 936 \\
\hline $\operatorname{Sex}(M / F)$ & $978: 652$ & $638: 426$ & $765: 507$ & $383: 473$ & $288: 198$ & $688: 248$ \\
\hline Age $(\text { years })^{\mathrm{a}}$ & $61.5 \pm 11.5$ & $45.5 \pm 9.5$ & $65.0 \pm 9.5$ & $69.5 \pm 6.8$ & $64.2 \pm 11.5$ & $52.1 \pm 14.6$ \\
\hline BMI $\left(\mathrm{kg} / \mathrm{m}^{2}\right)^{\mathrm{a}}$ & $23.7 \pm 3.9$ & $22.7 \pm 3.0$ & $24.2 \pm 3.8$ & $23.8 \pm 3.7$ & $24.9 \pm 4.6$ & $23.6 \pm 3.2$ \\
\hline $\mathrm{FPG}(\mathrm{mmol} / \mathrm{l})^{\mathrm{a}}$ & $9.1 \pm 3.5$ & $5.2 \pm 1.1$ & $8.9 \pm 3.0$ & $5.1 \pm 0.7$ & $7.4 \pm 2.1$ & NA \\
\hline $\mathrm{HbA}_{1 \mathrm{c}}(\%)^{\mathrm{a}}$ & $7.4 \pm 1.6$ & $4.7 \pm 0.4$ & $7.3 \pm 1.6$ & $5.2 \pm 0.2$ & $6.7 \pm 1.0$ & NA \\
\hline Duration of diabetes (years) ${ }^{\mathrm{a}}$ & $10.8 \pm 9.2$ & - & $13.4 \pm 9.7$ & - & $15.9 \pm 14.6$ & - \\
\hline
\end{tabular}

${ }^{\mathrm{a}}$ Mean $\pm \mathrm{SD}$

F, female; FPG, fasting plasma glucose; M, male; NA, not available

Table 2 Genotype and allele frequencies of candidate SNP loci in the three Japanese populations

\begin{tabular}{|c|c|c|c|c|}
\hline \multirow[t]{2}{*}{$\mathrm{SNP}^{\mathrm{a}}$} & \multicolumn{2}{|l|}{ Cases (type 2 diabetes) } & \multicolumn{2}{|l|}{ Controls } \\
\hline & Risk-allele frequency $(11 / 12 / 22)^{\mathrm{b}}$ & $p$ value for $\mathrm{HWE}$ & Risk-allele frequency $(11 / 12 / 22)^{\mathrm{b}}$ & $p$ value for HWE \\
\hline \multicolumn{5}{|c|}{ rs864745 ( $\underline{\mathrm{T}}>\mathrm{C}), J A Z F 1, \mathrm{Chr} 7$} \\
\hline Set 1 & $0.796(1,009 / 522 / 64)$ & 0.73 & $0.785(612 / 320 / 52)$ & 0.23 \\
\hline Set 2 & $0.797(793 / 411 / 49)$ & 0.64 & $0.793(532 / 262 / 42)$ & 0.19 \\
\hline Set 3 & $0.813(310 / 136 / 19)$ & 0.41 & $0.774(557 / 321 / 49)$ & 0.76 \\
\hline \multicolumn{5}{|c|}{ rs12779790 (A> $\underline{\mathrm{G}}), C D C 123 / C A M K 1 D$, Chr 10} \\
\hline Set 1 & $0.172(1,101 / 457 / 48)$ & 0.94 & $0.149(718 / 244 / 25)$ & 0.44 \\
\hline Set 2 & $0.164(855 / 343 / 30)$ & 0.52 & $0.148(566 / 202 / 15)$ & 0.54 \\
\hline Set 3 & $0.144(348 / 122 / 8)$ & 0.47 & $0.144(683 / 216 / 25)$ & 0.12 \\
\hline \multicolumn{5}{|c|}{ rs7961581 ( $>\underline{C}), T S P A N 8 / L G R 5$, Chr 12} \\
\hline Set 1 & $0.221(966 / 540 / 81)$ & 0.62 & $0.213(606 / 317 / 49)$ & 0.37 \\
\hline Set 2 & $0.216(777 / 418 / 63)$ & 0.49 & $0.193(553 / 252 / 36)$ & 0.29 \\
\hline Set 3 & $0.230(273 / 167 / 23)$ & 0.69 & $0.208(581 / 306 / 40)$ & 0.97 \\
\hline \multicolumn{5}{|c|}{ rs4607103 (두), ADAMTS9, Chr 3} \\
\hline Set 1 & $0.618(612 / 739 / 238)$ & 0.54 & $0.599(346 / 492 / 151)$ & 0.27 \\
\hline Set 2 & $0.621(469 / 622 / 165)$ & 0.06 & $0.620(317 / 402 / 116)$ & 0.52 \\
\hline Set 3 & $0.600(169 / 236 / 73)$ & 0.53 & $0.612(368 / 406 / 159)$ & 0.01 \\
\hline \multicolumn{5}{|c|}{ rs10923931 (G>T $)$, NOTCH2, Chr 1} \\
\hline Set 1 & $0.019(1,529 / 60 / 0)$ & 0.44 & $0.017(954 / 33 / 0)$ & 0.59 \\
\hline Set 2 & $0.017(1,222 / 42 / 1)$ & 0.31 & $0.017(816 / 27 / 1)$ & 0.13 \\
\hline Set 3 & $0.014(466 / 13 / 0)$ & 0.76 & $0.021(897 / 36 / 2)$ & 0.01 \\
\hline \multicolumn{5}{|c|}{ rs1153188 ( $\underline{\mathrm{A}}>\mathrm{T}), D C D$, Chr 12} \\
\hline Set 1 & $0.971(1,510 / 86 / 3)$ & 0.13 & $0.982(947 / 36 / 0)$ & 0.56 \\
\hline Set 2 & $0.979(1,215 / 49 / 2)$ & 0.05 & $0.982(808 / 30 / 0)$ & 0.60 \\
\hline Set 3 & $0.984(454 / 15 / 0)$ & 0.72 & $0.985(903 / 28 / 0)$ & 0.64 \\
\hline \multicolumn{5}{|c|}{ rs9472138 (C> T), VEGFA, Chr 6} \\
\hline Set 1 & $0.119(1,259 / 325 / 29)$ & 0.14 & $0.095(808 / 173 / 7)$ & 0.49 \\
\hline Set 2 & $0.110(1,009 / 234 / 22)$ & 0.05 & $0.099(678 / 154 / 6)$ & 0.39 \\
\hline Set 3 & $0.082(407 / 69 / 5)$ & 0.29 & $0.106(744 / 181 / 8)$ & 0.41 \\
\hline
\end{tabular}

${ }^{\mathrm{a}}$ The underlined allele represents the risk allele reported in European populations

${ }^{\mathrm{b}} 11$, homozygous for major allele; 12, heterozygous; 22, homozygous for minor allele

Chr, chromosome 
general population registered in the Japanese singlenucleotide polymorphism (SNP) database as Controls (Control 3; $n=936,688$ men and 248 women, Table 1).

The clinical characteristics of the participants are summarised in Table 1. Diabetes was diagnosed according to the WHO criteria [18]. Type 2 diabetes is clinically defined as a disease with gradual adult onset. Individuals who tested positive for anti-GAD antibodies and those diagnosed as having mitochondrial disease (mitochondrial myopathy, encephalopathy, lactic acidosis and stroke-like episodes) or maturity-onset diabetes of the young were not included in the Case patient groups. Written informed consent was obtained from all the participants, and DNA was extracted using the standard phenol-chloroform procedure. The protocol was approved by the ethics committee of the RIKEN Yokohama Institute, Japan.

In the present study, we first chose the 11 SNPs that emerged in stage 2 of the GWAS meta-analysis for type 2 diabetes in European populations [14], and selected seven of them (rs864745 in JAZF1, rs12779790 near CDC123/ CAMK1D, rs7961581 near TSPAN8/LGR5, rs4607103 near ADAMTS9, rs10923931 in NOTCH2, rs1153188 near DCD, and rs9472138 near VEGFA) to investigate their association with type 2 diabetes in several independent Japanese populations. With regard to the remaining four SNPs, one (rs 17036101) is in high linkage disequilibrium (LD) $\left(r^{2}=0.8\right)$ with rs1801282 (Pro12Ala polymorphism in PPARG), two SNPs (rs7578597 and rs10490072) have been shown to be mono-allelic in the Japanese, and one (rs2641348) is within absolute LD to rs10923931 in NOTCH2. Therefore, these four SNPs were excluded from the present study. Each SNP genotyping was performed by the TaqMan assay (Applied Biosystems, Foster City, CA, USA). The success rates of these assays were $>95 \%$ and the concordance rates in selected duplicate samples (1.5\% of all study individuals) were 99.8\% (rs864745, 98.8\%; rs $12779790,100 \%$; rs7961581, 100\%; rs4607103, 100\%; rs10923931, $100 \%$; rs1153188, 100\%; rs9472138, 100\%).

Statistical analysis The power of sample size to identify the association of investigated SNPs with type 2 diabetes was calculated using 'CaTS power calculator for genetic studies' software (www.sph.umich.edu/csg/abecasis/CaTS/, accessed 1 November 2008). We performed Hardy-Weinberg equilibrium (HWE) tests according to the method described by Nielsen et al. [19]. To evaluate the difference in the population structure among Case and Control groups, we performed Wright's $F$ statistics [20]. Individuals randomly selected from each group (about 376 individuals each) were genotyped for 43 randomly selected SNPs, and the results indicated that there is little difference in population structure between the present Case and Control groups (Electronic supplementary material [ESM] Table 1).
The differences between the Case and Control groups in terms of distribution of genotype scored with an additive model were evaluated using a logistic regression analysis, adjusting for sex, age and $\log _{e}$-transformed BMI. We further performed a meta-analysis with a fixed-effect model

Table 3 Association of candidate SNP loci with type 2 diabetes

\begin{tabular}{|c|c|c|c|}
\hline $\mathrm{SNP}^{\mathrm{a}}$ & $\mathrm{OR}^{\mathrm{b}}$ & $95 \% \mathrm{CI}$ & $p$ value ${ }^{\mathrm{c}}$ \\
\hline \multicolumn{4}{|c|}{ rs864745 ( $\underline{\mathrm{T}}>\mathrm{C}), J A Z F 1, \mathrm{Chr} 7$} \\
\hline Set 1 & 1.172 & $0.979-1.404$ & 0.0844 \\
\hline Set 2 & 1.050 & $0.895-1.231$ & 0.5495 \\
\hline Set 3 & 1.315 & $1.058-1.636$ & 0.0137 \\
\hline Meta-analysis & 1.148 & $1.034-1.275$ & 0.0098 \\
\hline \multicolumn{4}{|c|}{ rs12779790 (A> $\underline{\mathrm{G}}), C D C 123 / C A M K 1 D$, Chr 10} \\
\hline Set 1 & 1.187 & $0.973-1.449$ & 0.0918 \\
\hline Set 2 & 1.081 & $0.900-1.300$ & 0.4054 \\
\hline Set 3 & 1.071 & $0.835-1.374$ & 0.5878 \\
\hline Meta-analysis & 1.115 & $0.990-1.256$ & 0.0729 \\
\hline \multicolumn{4}{|c|}{ rs7961581 ( $>\underline{\mathrm{C}})$, TSPAN8/LGR5, Chr 12} \\
\hline Set 1 & 0.986 & $0.825-1.178$ & 0.8784 \\
\hline Set 2 & 1.188 & $1.015-1.395$ & 0.0321 \\
\hline Set 3 & 1.118 & $0.900-1.389$ & 0.3133 \\
\hline Meta-analysis & 1.099 & $0.991-1.221$ & 0.0730 \\
\hline \multicolumn{4}{|c|}{ rs4607103 ( $\underline{\mathrm{C}}>\mathrm{T})$, ADAMTS9, Chr 3} \\
\hline Set 1 & 1.049 & $0.901-1.221$ & 0.5353 \\
\hline Set 2 & 0.988 & $0.863-1.133$ & 0.8606 \\
\hline Set 3 & 1.037 & $0.866-1.240$ & 0.6951 \\
\hline Meta-analysis & 1.020 & $0.933-1.114$ & 0.6644 \\
\hline \multicolumn{4}{|c|}{ rs10923931 (G> T), NOTCH2, Chr 1} \\
\hline Set 1 & 0.942 & $0.529-1.679$ & 0.8390 \\
\hline Set 2 & 1.029 & $0.637-1.685$ & 0.9078 \\
\hline Set 3 & 0.630 & $0.321-1.236$ & 0.1786 \\
\hline Meta-analysis & 0.892 & $0.645-1.235$ & 0.4926 \\
\hline \multicolumn{4}{|c|}{ rs1153188 $(\underline{\mathrm{A}}>\mathrm{T}), D C D$, Chr 12} \\
\hline Set 1 & 0.499 & $0.300-0.831$ & 0.0076 \\
\hline Set 2 & 0.902 & $0.560-1.429$ & 0.6637 \\
\hline Set 3 & 1.014 & $0.492-2.092$ & 0.9697 \\
\hline Meta-analysis & 0.740 & $0.542-1.009$ & 0.0571 \\
\hline \multicolumn{4}{|c|}{ rs9472138 (C>T), VEGFA, Chr 6} \\
\hline Set 1 & 1.243 & $0.974-1.587$ & 0.0803 \\
\hline Set 2 & 1.068 & $0.868-1.318$ & 0.5342 \\
\hline Set 3 & 0.811 & $0.594-1.106$ & 0.1858 \\
\hline Meta-analysis & 1.062 & $0.922-1.223$ & 0.4074 \\
\hline
\end{tabular}

Meta-analysis was performed with a fixed-effect model after investigating evidence of heterogeneity

${ }^{\mathrm{a}}$ The underlined allele represents the risk allele reported in European populations

${ }^{\mathrm{b}}$ OR for original risk alleles reported in European populations

${ }^{\mathrm{c}} p$ values were calculated using a logistic regression analysis with the additive model (adjusted for sex, age and $\log _{e}$-transformed BMI)

Chr, chromosome 
after investigating evidence for heterogeneity of the ORs using Cochran's $Q$ statistics [14, 21]. Combined estimates of the ORs were calculated by weighting the $\log _{e}$ ORs of each study by the inverse of their variance.

\section{Results}

The genotype distribution of each SNP selected for the present study (see Methods) in the Case and Control groups was shown in Table 2. Genotype distributions of the most SNP loci did not deviate from HWE, except for two SNPs (rs4607103 and rs10923931) whose distributions showed borderline values in Control 3 ( $p=0.01$, Table 2).

As shown in Table 3, rs864745 and rs12779790 tend to have the same direction of the association with type 2 diabetes in all three populations, as previously reported in European GWASs. Subsequent meta-analyses revealed that rs864745 showed a nominal association with susceptibility to type 2 diabetes (nominal $p=0.0098$, OR 1.148, 95\% CI 1.034-1.275), but the association did not stand after Bonferroni's correction (corrected $p=0.068$ ). We could not observe any association between other SNP loci and type 2 diabetes (nominal $p>0.05$ ). Among seven SNP loci examined in this study, five showed the same direction as the direction initially reported by Zeggini et al. [14], and a probability that five or more SNPs go in the same direction is 0.23 $\left(\left[{ }_{7} C_{5}=21\right] \times 0.5^{5} \times 0.5^{2}+\left[{ }_{7} C_{6}=7\right] \times 0.5^{6} \times 0.5^{1}+{ }_{7} C_{7}=1\right]$ $\left.\times 0.5^{7} \times 0.5^{0}\right)$ based on a binomial distribution.

We further compared frequencies of the reported risk allele in the Japanese populations with those in the European populations (Table 4). The allele frequencies for most of the SNPs examined in the present study were considerably different between the Japanese populations and populations of European descent.

\section{Discussion}

In the present study, in three independent Japanese population sets we examined seven additional SNPs that emerged in stage 2 of the GWAS meta-analysis for type 2 diabetes in European populations. We demonstrated a nominal association of rs 864745 in $J A Z F 1$ with the disease by a meta-analysis, but the association of all seven SNP loci did not attain statistically significant levels after correction for multiple testing errors.

Recent advances in human genetic research have facilitated the identification of genes conferring susceptibility to common diseases such as type 2 diabetes from across the entire human genome by using a large number of individuals, and GWASs have been conducted worldwide [3-9]. The latest meta-analysis of GWASs for type 2 diabetes in European populations has reported the existence of 11 candidate SNPs. Among them, six SNP loci were confirmed as new susceptibility loci for type 2 diabetes in those of European descent through large-scale replication studies [14].

In our meta-analysis, one SNP, rs864745 in JAZF1, among the examined seven SNPs had nominal association with type 2 diabetes in the Japanese populations with the same direction as previously reported (Table 3) [14], but the association of all seven SNPs, including rs864745, did not reach statistically significant levels after Bonferroni's correction (corrected $p>0.05$ ). Since the Control individuals were significantly younger than the Case patients in our first and third Sets (Table 1), we further selected Control participants who were $\geq 50$ years in these Sets (ESM Tables 2 and 3). However, the results were not changed after we re-analysed the association of the SNP loci with type 2 diabetes using these selected Control individuals by logistic regression analyses followed by meta-analyses (ESM Table 4).

Table 4 Comparison of risk-allele frequencies in the Japanese populations with those in European populations

\begin{tabular}{|c|c|c|c|c|c|c|}
\hline \multirow[t]{2}{*}{ SNP } & \multirow[t]{2}{*}{ Risk allele $^{\mathrm{a}}$} & \multirow[t]{2}{*}{ Nearest gene } & \multicolumn{3}{|c|}{ Japanese populations } & \multirow[t]{2}{*}{ European populations ${ }^{\mathrm{b}}$} \\
\hline & & & Control 1 & Control 2 & Control 3 & \\
\hline rs864745 & $\mathrm{T}$ & $J A Z F 1$ & 78.5 & 79.3 & 77.4 & 50.1 \\
\hline rs12779790 & $\mathrm{G}$ & $C D C 123 / C A M K 1 D$ & 14.9 & 14.8 & 14.4 & 18.3 \\
\hline rs7961581 & $\mathrm{C}$ & TSPAN8/LGR5 & 21.3 & 19.3 & 20.8 & 26.9 \\
\hline rs4607103 & $\mathrm{C}$ & ADAMTS9 & 59.9 & 62.0 & 61.2 & 76.1 \\
\hline rs10923931 & $\mathrm{T}$ & $\mathrm{NOTCH} 2$ & 1.7 & 1.7 & 2.1 & 10.6 \\
\hline rs1153188 & A & $D C D$ & 98.2 & 98.2 & 98.5 & 73.3 \\
\hline rs9472138 & $\mathrm{T}$ & VEGFA & 9.5 & 9.9 & 10.6 & 28.2 \\
\hline
\end{tabular}

\footnotetext{
${ }^{\text {a }}$ Risk allele in the European populations

${ }^{\mathrm{b}}$ Sample-size weighted risk-allele frequencies across the DGI/FUSION/UK stage 2 studies, previously reported by Zeggini et al. [14]
} 
The discrepancy of the results between the present study and the European study reported by Zeggini et al. [14] may be explained by the ethnic difference, because clear differences in the allele frequencies of these SNPs exist between the Japanese and European populations (Table 4). The genotype distributions of all examined SNPs in the present study were considered to be within HWE, although those for rs4607103 and rs10923931 showed borderline values in Control 3 (Table 2). Because the concordance rate for the present study was nearly $100 \%$, and the population structures of our Case and Control groups were shown to be the same by Wright's $F$ statistics (ESM Table 1), it is unlikely that our present data were skewed by the genotyping errors or by the presence of population stratification. However, considering the relatively weak effects of these loci, ORs were approximately 1.1 in European populations [14], and the estimated powers of the present study were $61 \%, 56 \%, 67 \%, 78 \%, 14 \%, 11 \%$ and $43 \%$ at $\alpha<0.05$, and $33 \%, 29 \%, 39 \%, 51 \%, 3 \%, 3 \%$ and $19 \%$ at $\alpha<0.0071$ (significance level after Bonferroni's adjustment) for rs864745, rs12779790, rs7961581, rs4607103, rs10923931, rs1153188 and rs9472138, respectively; thus, the possibility of insufficient study power could also be a probable reason for missing these SNPs as susceptibility variants for type 2 diabetes.

Recently, Grarup et al. reported that three SNP loci, namely, rs864745 in JAZF1, rs12779790 and rs7961581, were significantly associated with traits of insulin secretion in a glucose-tolerant Danish population [22]. Since rs12779790 near CDC123/CAMK1D and rs7961581 near TSPAN8/LGR5, in addition to rs864745, tended to show borderline association with type 2 diabetes in our metaanalysis (nominal $p=0.0729$ and 0.0730 , respectively, Table 3), these two SNP loci in addition to rs 864745 might have some effects on conferring susceptibility to type 2 diabetes also in Japanese populations. However, another similar study by Staiger et al. was unable to demonstrate any significant association of these SNPs with prediabetic traits [23]. Therefore, further study will be required in the Japanese as well as in other ethnic populations to evaluate the association of those 11 SNP loci, including six SNPs confirmed by Zeggini et al. [14] with type 2 diabetes.

In conclusion, we performed a replication study for the association of the meta-analysis-derived risk loci for type 2 diabetes in 6,244 Japanese individuals from three independent populations, and identified that rs864745 in JAZF1 showed a nominal association with type 2 diabetes, although the association of all seven SNPs in this study did not attain statistically significant levels. Therefore, the contribution of the seven examined loci in conferring susceptibility to type 2 diabetes is considered minor in the Japanese population, if they are present.
Acknowledgements We thank K. Hotta, S. Tsukada and H. Unoki at the Laboratory for Endocrinology and Metabolism, RIKEN Center for Genomic Medicine for helpful discussions and thank the technical staff of the Laboratory for Endocrinology and Metabolism, RIKEN Center for Genomic Medicine, for their technical assistance. This work was partly supported by a Grant-in-Aid from the Ministry of Education, Culture, Sports, Science and Technology, Japan (to S. Maeda).

Duality of interest The authors declare that there is no duality of interest associated with this manuscript.

\section{References}

1. O'Rahilly S, Barroso I, Wareham NJ (2005) Genetic factors in type 2 diabetes: the end of the beginning? Science 307:370373

2. Grant SF, Thorleifsson G, Reynisdottir I et al (2006) Variant of transcription factor 7-like 2 (TCF7L2) gene confers risk of type 2 diabetes. Nat Genet 38:320-323

3. Sladek R, Rocheleau G, Rung J et al (2007) A genome-wide association study identifies novel risk loci for type 2 diabetes. Nature 445:881-885

4. Zeggini E, Weedon MN, Lindgren CM et al (2007) Replication of genome-wide association signal in UK samples reveals risk loci for type 2 diabetes. Science 316:1336-1341

5. Scott LJ, Mohlke KL, Bonnycastle LL et al (2007) A genomewide association study of type 2 diabetes in Finns detects multiple susceptibility variants. Science 316:1341-1345

6. Saxena R, Voight BF, Lyssenko V et al (2007) Genome-wide association analysis identifies loci for type 2 diabetes and triglyceride levels. Science 316:1331-1336

7. Steinthorsdottir V, Thorleifsson G, Reynisdottir I et al (2007) A variant in CDKAL1 influences insulin response and risk of type 2 diabetes. Nat Genet 39:770-775

8. Unoki H, Takahashi A, Kawaguchi T et al (2008) SNPs in $K C N Q 1$ are associated with susceptibility to type 2 diabetes in East Asian and European populations. Nat Genet 40:1098-1102

9. Yasuda K, Miyake K, Horikawa Y et al (2008) Variants in KCNQ1 are associated with susceptibility to type 2 diabetes mellitus. Nat Genet 40:1092-1097

10. Omori S, Tanaka Y, Takahashi A et al (2008) Association of CDKAL1, IGF2BP2, CDKN2A/B, HHEX, SLC30A8, and KCNJ11 with susceptibility to type 2 diabetes in a Japanese population. Diabetes 57:791-795

11. Horikoshi M, Hara K, Ito C et al (2007) Variations in the HHEX gene are associated with increased risk of type 2 diabetes in the Japanese population. Diabetologia 50:2461-2466

12. Furukawa Y, Shimada T, Furuta $\mathrm{H}$ et al (2008) Polymorphisms in the IDE-KIF11-HHEX gene locus are reproducibly associated with type 2 diabetes in a Japanese population. J Clin Endocrinol Metab 93:310-314

13. Horikawa Y, Miyake K, Yasuda K et al (2008) Replication of genome-wide association studies of type 2 diabetes susceptibility in Japan. J Clin Endocrinol Metab 93:3136-3141

14. Zeggini E, Scott LJ, Saxena R et al (2008) Meta-analysis of genome-wide association data and large-scale replication identifies additional susceptibility loci for type 2 diabetes. Nat Genet 40:638-645

15. Maeda S, Tsukada S, Kanazawa A et al (2005) Genetic variations in the gene encoding TFAP $2 B$ are associated with type 2 diabetes mellitus. J Hum Genet 50:283-292 
16. Hayashi T, Iwamoto Y, Kaku K, Hirose H, Maeda S (2007) Replication study for the association of TCF $7 L 2$ with susceptibility to type 2 diabetes in a Japanese population. Diabetologia 50:980-984

17. The International HapMap Consortium (2005) A haplotype map of the human genome. Nature 437:1299-1320

18. Alberti KG, Zimmet PZ (1998) Definition, diagnosis and classification of diabetes mellitus and its complications. Part 1: diagnosis and classification of diabetes mellitus provisional report of a WHO consultation. Diabet Med 15:539-553

19. Nielsen DM, Ehm MG, Weir BS (1998) Detecting marker-disease association by testing for Hardy-Weinberg disequilibrium at a marker locus. Am J Hum Genet 63:1531-1540
20. Wright S (1969) Evolution and the genetics of populations, volume 2: the theory of gene frequencies. University of Chicago Press, Chicago, IL, pp 294-295

21. Cochran WG (1954) The combination of estimates from different experiments. Biometrics 10:101-129

22. Grarup N, Andersen G, Krarup NT et al (2008) Association testing of novel type 2 diabetes risk-alleles in the JAZF1, CDC123/CAMK1D, TSPAN8, THADA, ADAMTS9, and NOTCH2 loci with insulin release, insulin sensitivity and obesity in a population-based sample of 4, 516 glucose-tolerant middle-aged Danes. Diabetes 57:2534-2540

23. Staiger H, Machicao F, Kantartzis K et al (2008) Novel metaanalysis-derived type 2 diabetes risk loci do not determine prediabetic phenotypes. PLoS ONE 3:e3019 